\section{Intraoperative optical coherence tomography: past, present, and future \\ JP Ehlers}

\author{
Abstract \\ To provide an overview of the current \\ state of intraoperative optical coherence \\ tomography (OCT). Literature review of \\ studies pertaining to intraoperative OCT \\ examining both the technology aspects of \\ the imaging platform and the current \\ evidence for patient care. Over the last \\ several years, there have been significant \\ advances in integrative technology for \\ intraoperative OCT. This has resulted in \\ the development of multiple microscope- \\ integrated systems and a rapidly expanding \\ field of image-guided surgical care. \\ Multiple studies have demonstrated the \\ potential role for intraoperative OCT in \\ facilitating surgeon understanding of the \\ surgical environment, tissue configuration, \\ and overall changes to anatomy. In fact, \\ the PIONEER and DISCOVER studies, \\ both demonstrated a potential significant \\ percentage of cases that intraoperative OCT \\ alters surgical decision-making in both \\ anterior and posterior segment surgery. \\ Current areas of exploration and develop- \\ ment include OCT-compatible instrumenta- \\ tion, automated tracking, intraoperative \\ OCT software platforms, and surgeon \\ feedback/visualization platforms. Intra- \\ operative OCT is an emerging technology \\ that holds promise for enhancing the \\ surgical care of both anterior segment and \\ posterior segment conditions. Hurdles \\ remain for adoption and widespread \\ utilization, including cost, optimized \\ feedback platforms, and more definitive \\ value for individualized surgical care with \\ image guidance. \\ Eye (2016) 30, 193-201; doi:10.1038/eye.2015.255; \\ published online 18 December 2015
}

\section{Introduction}

In 1962, Thomas Kuhn coined the term 'paradigm shift' and defined it as an advancement in a field often defined by disruptive changes in methods, concepts or systems. ${ }^{1}$ Ophthalmic surgery has experienced numerous paradigm shifts over the past several decades. In the 1960s and 1970s, new surgical systems, such as vitrectomy and phacoemulsification revolutionized anterior segment and vitreoretinal surgery. ${ }^{2}$ In addition, visualization advances occurred in surgical microscopy, including foot-pedal control, hands-free manipulation, and wide-angle viewing systems. ${ }^{3}$ Yet with all these advances, new cutting-edge platforms for surgical visualization are still lacking.

From the cornea to the retina to the optic nerve, optical coherence tomography (OCT) has markedly impact clinicians' approach to diagnosis, management, and surveillance of ophthalmic diseases. ${ }^{4,5}$ Imaging has now become ubiquitous throughout patient care. However, even in the midst of this digital revolution the application of this technology to the operating room has been slow. Optical coherence tomography provides high-resolution cross-sectional and tomographic data. Using this technology, surgeons would potentially have immediate feedback on completion of surgical objectives or new understanding of the anatomic configuration of the tissues. If used real time, OCT could provide specific guidance for surgical manipulations.

Over the past several years, the introduction of OCT into the OR has resulted in new insights into the potential roles of this technology in the surgical management of ophthalmic conditions. This review will focus on the progress in intraoperative OCT technology and potential future directions for the field.

\section{Hurdles to utilization}

An initial barrier for bringing OCT to the operating room was the lack of integrative solutions. Standard desktop systems lacked portability. In addition, the traditional chin-rest approach for image acquisition is not practical
Ophthalmic Imaging Center, Cole Eye Institute, Cleveland Clinic, Cleveland, OH, USA

Correspondence:

JP Ehlers, Cole Eye Institute, Cleveland Clinic,

Foundation, 9500 Euclid

Ave/i32, Cleveland, 44195,

$\mathrm{OH}, \mathrm{USA}$

Tel: +1 216 6360183;

Fax: +12164452226

E-mail: ehlersj@ccf.org

Received: 12 October 2015 Accepted: 13 October 2015 Published online:

18 December 2015

Portions presented at the 45th Cambridge Ophthalmological Symposium. 
for a supine patient under sterile conditions. The general costs associated with intraoperative OCT are also a potential barrier. The OCT engine and system itself represent a significant investment. Alterations to surgical flow, such as potential delays in operating time, also may result in increased cost. Intraoperative OCT software applications are also needed to facilitate optimal interpretation and utilization of the data obtained during surgery. Finally, a limited understanding of the clinical applications of intraoperative OCT, as well as the overall value for outcomes has been a limiting factor for adoption.

\section{Intraoperative OCT systems and devices}

Standard clinical OCT systems are large and stationary, limiting their portability for operating room use. Modifications to the these table top systems can enable intraoperative imaging. However, this is logistically difficult making this approach impractical.

Portable OCT systems were the first major transformational step in intraoperative OCT. Two primary portable systems have been described in the literature: the Bioptigen EnVisu (Bioptigen, Research Triangle Park, NC/Leica, Wetzlar, Germany) and the Optovue iVue (Optovue, Fremont, CA, USA). ${ }^{6-17}$ Multiple approaches to image acquisition can be used with these systems, including handheld, external mounting, and microscope mounting.

Handheld imaging was the first described imaging approach with these systems. ${ }^{6-9,18}$ Although handheld imaging may provide excellent image quality, there are significant challenges that must be considered. Handheld imaging limits image reproducibility and optimal aiming. Motion artifacts owing to limited stability can delay image acquisition and impact image quality. Maintaining a sterile surgical field may also be particularly challenging using handheld imaging.

To address many of the limitations of handheld imaging, mounting systems were developed for these portable OCT systems. ${ }^{11,13-17}$ External mounts provide improved stability but introduce an additional footprint and space requirement to the operating room. Microscope mounts allow the surgeon to fasten the portable probe directly to the microscope body (Figure 1). With the probe attached to the microscope, increased stability is achieved compared with handheld imaging. In addition, the foot-pedal controls of the microscope enable $X-Y-Z$ foot-pedal control of the probe location. This facilitates rapid imaging with enhanced image reproducibility. ${ }^{13}$

Although portable and microscope-mounted systems are able to obtain images of excellent quality and provide surgeon feedback, all of these early systems require the surgeon to cease surgery before obtaining imaging. Although these delays have been reported to be quite minimal, a more seamless approach is needed to allow for 'real-time' imaging and minimize any surgical delay while obtaining imaging. ${ }^{13}$
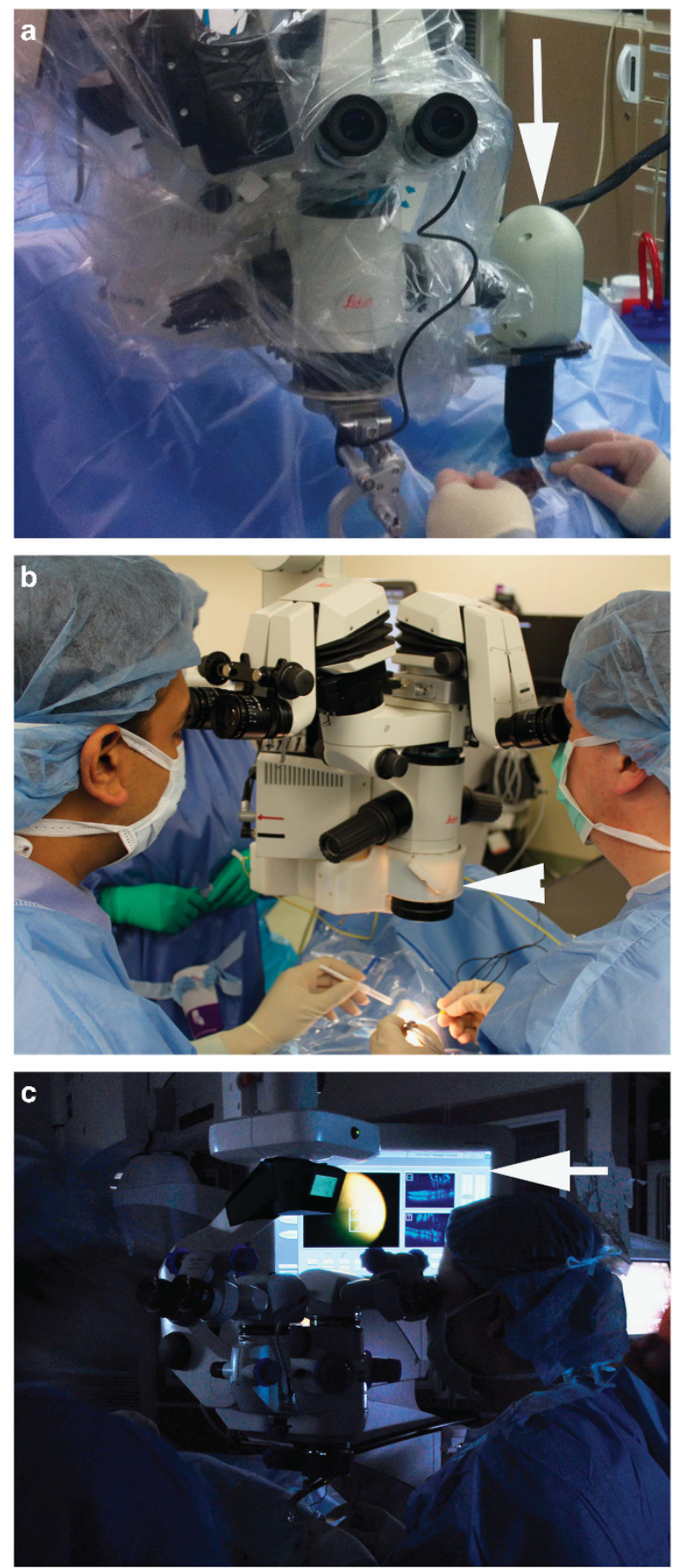

Figure 1 Intraoperative OCT systems. (a) Bioptigen microscopemounted portable OCT system (arrow). (b) Cole Eye microscopeintegrated OCT system (arrowhead) mounted between the microscope body and the microscope objective lens. (c) Zeiss Rescan 700 microscope-integrated OCT system. Display panel (arrow) showing both surgical view and OCT data stream. 
The next major advance in intraoperative OCT systems was the integration of the OCT and microscope optics. Adaptation of both Leica (Leica, Wetzlar, Germany) and Zeiss (Carl Zeiss Meditec, Oberkochen, Germany) were among the first systems described. ${ }^{19-21}$ The Zeiss adaptation used a modified Cirrus (Carl Zeiss Meditec) OCT system adapted for integration into the Zeiss microscope optical path. ${ }^{21}$ For anterior segment imaging, a modified Visante (Carl Zeiss Meditec) prototype was also integrated into a Zeiss microscope. ${ }^{22,23}$ Similarly, the Leica adaptation used a separate OCT scan head that was mounted to the bottom of the microscope body and integrated the optical paths of the two systems. ${ }^{19,20}$ These systems provided the first insights of the potential of 'real-time' intraoperative OCT allowing for OCT-based visualization of instrument-tissue interaction and immediate imaging with surgeon feedback. ${ }^{21,24}$ However, these systems also uncovered new challenges for intraoperative OCT development, including the need for new software systems, the limits of current instrumentation for OCT compatibility, and need for heads-up visualization. ${ }^{19,21}$ In addition, the need for $\mathrm{Z}$ axis stabilization to maximize image visualization during surgical maneuvers was identified owing to the intermittent vertical displacement that occurs during surgical maneuvers. Methods for automated tracking and/or surgeon directed maneuvering of the OCT beam to areas of interest were also lacking.

Following the development of the initial systems, second generation systems have now been developed, including the first commercially developed and approved systems. One system that has been described specifically addresses some of the shortcomings of early generation Leica add-on systems, including reduced form factor and decreased stack height (Figure 1). ${ }^{25,26} \mathrm{~A}$ tunable lens, heads-up display, and computer mouse-directed aiming provides this system with a more flexible and versatile approach to intraoperative imaging while minimizing the overall noticeable difference to the microscope footprint. ${ }^{25,26}$

In addition, three commercial systems have been described. The Zeiss Rescan 700 was the first FDA-cleared system and the second worldwide commercially available microscope-integrated intraoperative OCT system (Figure 1). ${ }^{27,28}$ The Rescan 700 is built on the Lumera 700 (Zeiss) microscope platform. The overall external form factor of the surgical microscope is unaltered compared with the Lumera system. The Rescan 700 combines the Callisto (Zeiss) external video control system to provide touchpad control of the OCT scanner and combined video/OCT visualization. The OCT system is also integrated into the microscope foot-pedal with surgeon directed control of aiming, orientation, and size of the OCT scan pattern. ${ }^{27,28}$ A heads-up display provides combined visualization of the surgical field and the OCT data stream. Anterior segment imaging and posterior segment imaging is feasible. The Rescan included one of the first Z-tracking systems for intraoperative OCT system, a significant advance for realtime intraoperative OCT. In its initial release, no analysis software was incorporated into the Rescan system and automated tracking was also not available. ${ }^{27,28}$

The Haag-Streit iOCT system (Haag-Streit, Koeniz, Switzerland) was the first globally commercially available and 2nd FDA-cleared microscope-integrated intraoperative OCT system. The Haag-Streit system uses a side port for OCT access using the OPMedT (OPMedT, Lubeck, Germany) OCT system, which is coupled to the microscope platform. ${ }^{29}$ The Haag-Streit system includes both a microscope-mounted viewing screen and a head-up display screen.

In addition to the commercially available system, Bioptigen has also developed a research prototype that may be adapted to either a Leica or Zeiss scope. The Bioptigen system has been tested with a variety of features, including extended range scanning and ultra high resolution. The system uses operator driven computer-directed OCT aiming. The long-fiber optic length provides increased flexibility for placement of the OCT engine and computer within the operating room. ${ }^{30}$

Next generation systems are currently under development. Previous systems have used spectral domain OCT technology. Swept source OCT provides a unique opportunity to address some of the current deficits in integrated OCT systems. ${ }^{31}$ The increased acquisition speed provides for visualization of real-time three-dimensional instrument-tissue interaction.

Real-time volumetric scanning at near video rates is possible given exceptional speed of the swept source system. Building on this technology, a heads-up display system has been developed that used three-dimensional technology to provide a unique surgeon feedback platform for surgeons. ${ }^{31}$

In addition, research is needed in platform optimization and surgeon feedback systems for optimal integration and utility. Technological advances in viewing systems, optics, digital technology, and interactive platforms will continue to push the envelope of opportunities for a true seamless surgical guidance system.

\section{Clinical use and impact}

Multiple smaller studies and large-scale studies have examined the role for intraoperative OCT in various clinical conditions, both anterior and posterior segment. ${ }^{6-9,11-18,21-23,27,28,32-41}$ The PIONEER study was a large prospective clinical study $(n=531)$ examining the role of intraoperative OCT with a microscope-mounted portable OCT system in ophthalmic surgery. ${ }^{13}$ The 
DISCOVER study is a subsequent prospective clinical study $(n=227)$ examining microscope-integrated OCT in ophthalmic surgery. ${ }^{27}$ Both of these studies along with several other smaller studies have helped to establish the potential clinical utility of this technology for multiple ophthalmic conditions and procedures.

\section{Anterior segment}

Intraoperative OCT has been performed for numerous anterior segment procedures, including lamellar keratoplasty, full-thickness penetrating keratoplasty, cataract surgery, corneal inlay procedures, and glaucoma surgical procedures. 9,12,13,22,23,27,29,32,39,41,42 Using intraoperative OCT surgeons are able to obtain immediate feedback on the status of the surgical procedure.

The PIONEER study included 275 eyes that underwent intraoperative OCT for anterior segment surgery. The majority of these (59\%) cases were lamellar keratoplasty procedures. ${ }^{13,43}$ The DISCOVER study included 91 eyes in the anterior segment arm of the study and $64 \%$ of eyes were enrolled for a lamellar keratoplasty procedure. ${ }^{27}$

Multiple studies, including the PIONEER and DISCOVER studies, have examined Descemet stripping automated endothelial keratoplasty (DSAEK) and Descemet membrane endothelial keratoplasty (DMEK) with intraoperative OCT. Graft orientation and position is easily visualized with intraoperative OCT. Subclinical interface fluid is also easily identified with intraoperative OCT (Figure 2). In fact, residual subclinical interface fluid at the end of the case has been associated with postoperative textural interface opacity. ${ }^{39}$ In the DISCOVER study, intraoperative OCT findings resulted in additional surgical maneuvers in $41 \%$ of patients. ${ }^{27}$ In the PIONEER study, intraoperative OCT identified residual persistent fluid in $48 \%$ of eyes that resulted in additional surgical maneuvers. In $18 \%$ of cases in the PIONEER study the surgeon believed there was residual fluid but intraoperative OCT confirmed complete apposition, mitigating the need for additional maneuvers. ${ }^{13}$ This highlights the potential discordance of surgeon perception from en face visualization and surgical reality in these procedures.

In DMEK, three studies have documented the utility of intraoperative OCT during this technically challenging procedure to date. ${ }^{27,29,41}$ Intraoperative OCT facilitates identification of graft orientation and appropriate unscrolling during graft manipulation. The DISCOVER study found that in $\sim 50 \%$ of cases the intraoperative OCT provided information that was discordant to surgeon impression. In addition, there is preliminary evidence that intraoperative OCT-guided DMEK may help facilitate the transition from DSAEK to DMEK for the novice DMEK surgeon. ${ }^{27}$

In deep anterior lamellar keratoplasty (DALK), multiple studies have reported improved visualization and understanding of dissection depth with intraoperative OCT. ${ }^{12,13,27,32}$ In fact, one study reported that dissection depth measured with intraoperative OCT appeared to be an important factor for successful DALK without
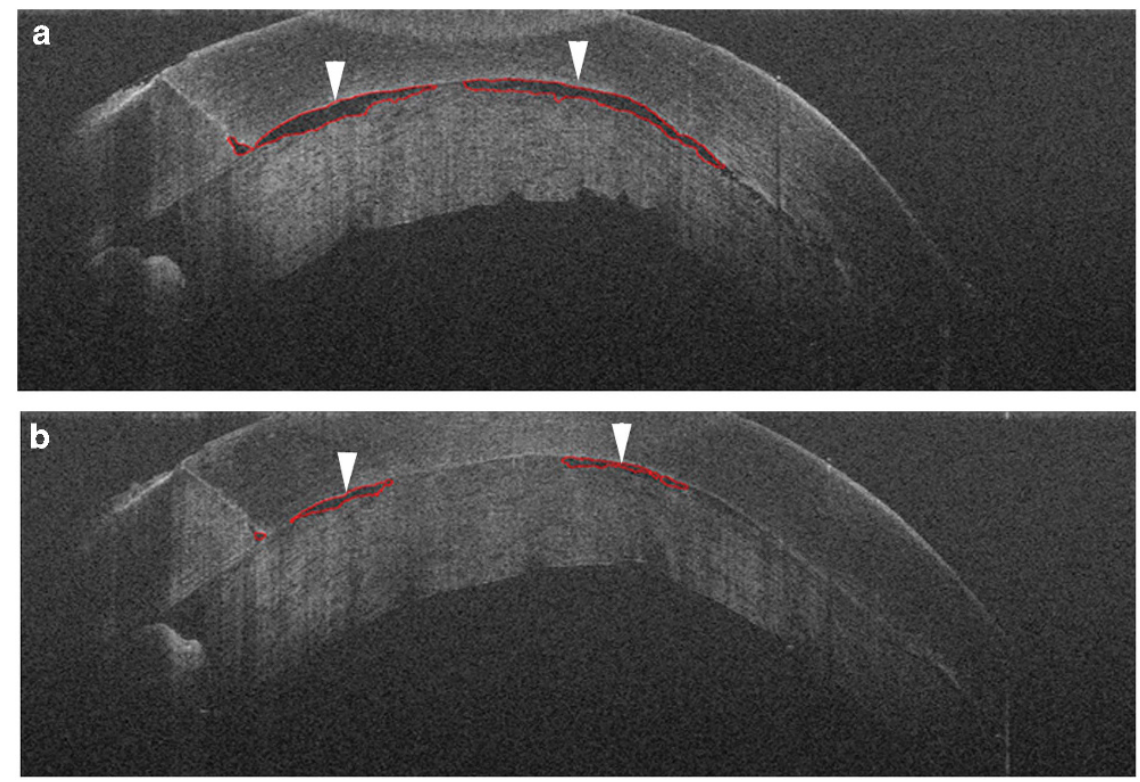

Figure 2 Intraoperative OCT for Descemet stripping automated endothelial keratoplasty with automated interface fluid analysis. (a) Time point 1 shows interface fluid between the graft and host (arrowheads) with automatic segmentation around fluid to evaluate overall fluid metrics (red lines). (b) Time point 2 shows reduction of interface fluid (arrowheads) following corneal manipulation with successful automated identification of fluid interface (red lines). 
conversion to full-thickness penetrating keratoplasty. ${ }^{12}$ The PIONEER and DISCOVER studies confirmed the potential role of intraoperative OCT in DALK. In some cases, intraoperative OCT identified subclinical big-bubble formation, which guided surgical maneuvers for successful dissection. In $38-56 \%$ of cases, intraoperative OCT facilitated changes in dissection depth. ${ }^{13,27}$

Using intraoperative OCT during cataract surgery facilitates confirmation of appropriate lens position, visualization of the corneal incision, and optimizes intraocular lens calculations. ${ }^{13,27}$ In fact, one study reported that intraoperative OCT-guided intraocular lens calculations are more accurate than preoperative assessment, presumably because of a more accurate identification of the post-operative lens position and calculation of anterior chamber depth. ${ }^{22}$

\section{Posterior segment}

For posterior segment surgical conditions, the potential for intraoperative OCT is expansive. Numerous conditions, including optic pit-related maculopathy, retinopathy of premature, macular hole, retinal detachment, proliferative diabetic retinopathy, epiretinal membrane, and posterior uveitis have been described with intraoperative OCT. $6-8,10,13-17,21,27,28,34,35,37,44,45$

In selected conditions, intraoperative OCT has provided new information on the underlying pathophysiology. In retinopathy of prematurity, preretinal structures and retinoschisis have been identified with intraoperative OCT that may markedly impact the surgical approach to these complex conditions. ${ }^{6}$ In optic pit-related maculopathy, intraoperative OCT demonstrated a potential connection between the macular schisis and the vitreous cavity. ${ }^{8}$ In macular hole cases, the post-peel expansion of the ellipsoid zone (EZ) to retinal pigment epithelium (RPE) height may be involved in the rate of anatomic normalization following surgical repair (Figure 3). ${ }^{17,34}$

In other cases, the direct and immediate feedback to surgeons can have a critical role in surgical decisionmaking and specific surgical manipulations. Membrane peeling, in particular, lends itself well to intraoperative OCT-assisted surgery. In both the PIONEER and DISCOVER study, there was a significant discordance between surgeon's microscope visualization of the pathology and the findings of the intraoperative OCT. ${ }^{13,27,30}$ In $13-22 \%$ of cases, intraoperative OCT identifies residual membranes that required membrane peeling that were otherwise not identified (Figure 3). ${ }^{13,27,30}$ Conversely, in cases where the surgeon believed there was more to peel, intraoperative OCT determined that in $15-40 \%$ of cases the surgical objectives had been completed and there was not any significant

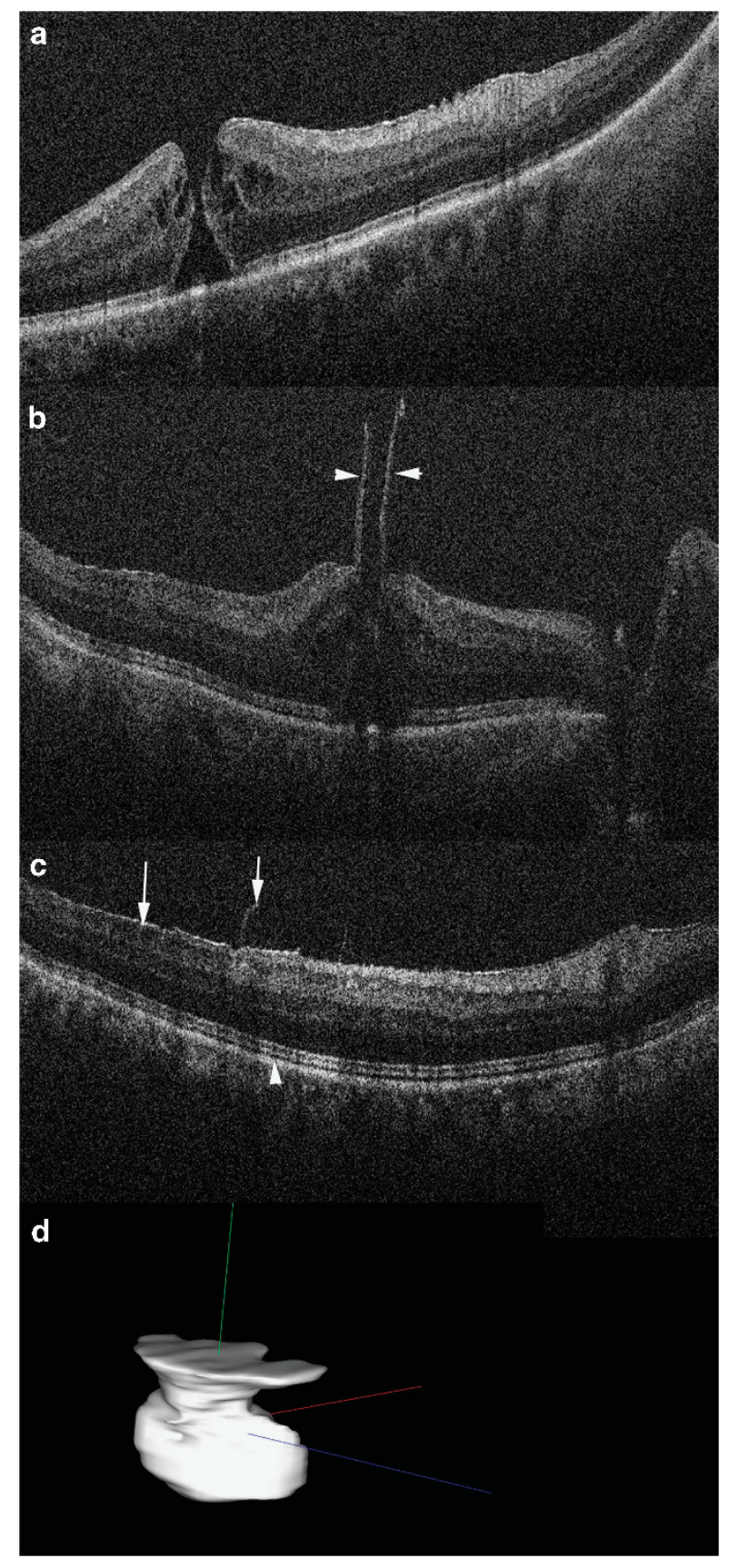

Figure 3 Intraoperative OCT for macular hole surgery. (a) Preincision OCT revealing full-thickness macular hole with minimal associated epiretinal membrane. (b) Post-peel OCT confirms large residual flap at edge of hole (arrowheads). Configuration information may be used to guide inverse internal limiting membrane flap technique. (c) Post-peel OCT also identified more peripheral residual epiretinal membrane edges (arrow) and expansion of the ellipsoid zone to retinal pigment epithelium height (arrowhead, compare with A). (d) Automated volumetric segmentation of macular hole with three-dimensional reconstruction allowing for visualization of alterations between pre- and post-peel configurations. 
residual membranes. ${ }^{13,27}$ Both macroarchitectural and microarchitectural changes have been described in the retina following surgical manipulations. ${ }^{46}$ In addition procedures where direct feedback has been documented includes subretinal perfluorocarbon liquid removal and subretinal injection of therapeutics. ${ }^{35}$ In addition, more subtle changes occur in the outer retina with expansion of the EZ-RPE height following membrane peeling.

In retinal detachment cases, subclinical persistent subretinal fluid under perfluorocarbon liquid tamponade has been identified in the vast majority of eyes undergoing surgical repair (Figure 4).13,27,35 In addition, foveal architectural changes, including occult full-thickness macular holes, have been visualized that may have prognostic implications (Figure 4). ${ }^{35}$ In the DISCOVER study, intraoperative OCT appeared to provide valuable information in $\sim 22 \%$ of cases, such as identification of optimal drainage sites and discriminating subretinal fluid from white without pressure. ${ }^{27}$

For proliferative diabetic retinopathy and vitreous hemorrhage cases, intraoperative OCT can provide important insights into the anatomic configuration of the tissues. ${ }^{14,27}$ Using a microscope-integrated system, intraoperative OCT facilitated tissue dissection by identification of surgical planes. In dense vitreous hemorrhage cases, intraoperative OCT identified a significant amount of underlying macular pathology that may potentially change intraoperative or postoperative management (for example, macular edema and intraretinal fluid). ${ }^{14}$

\section{Future needs and directions}

Although the last few years have seen landmark advances in the field of intraoperative OCT, continued progress is needed to overcome the current hurdles to seamless image-guided surgery. Several areas of need include OCT-compatible surgical instrumentation, automated tracking, software platforms, and a comprehensive surgeon feedback platform.

Current metallic surgical instrumentation creates difficulties for intraoperative OCT systems due to the light scattering and shadowing characteristics of metal. ${ }^{19,21,27}$ This limits visualization of tissue-instrument interactions with intraoperative OCT. Novel material testing and prototype development is underway to maximize OCT-based visualization whereas maintaining the surgical precision required for ophthalmic surgery. ${ }^{25}$ Alternatives to changes in materials could include software processing to minimize the overall impact of the instrument material on the visualization of the adjacent and underlying tissues, such as with spatial compounding. ${ }^{24}$

Automated tracking is an additional area of need to minimize surgeon burden for manual aiming to facilitate rapid imaging of the region of interest. ${ }^{47}$ Tracking could be performed for instrumentation and also for areas of interest on the retina away from surgical manipulation. Enhanced visualization through OCT-contrast agents, such as with triamcinolone or indocyanine green, may also be useful to improve tissue layer differentiation. 48,49

Software analysis platforms are currently lacking on commercial devices available for intraoperative OCT. However, studies have demonstrated the ability to analyze with automated algorithms subtle perturbations in the tissues following surgical manipulation. For example, in DSAEK, automated interface fluid segmentation and fluid mapping may provide important information regarding the maximal allowed fluid for surgical success or the optimal amount to reduce the risk of post-operative textural interface fluid (Figure 2). ${ }^{50} \mathrm{In}$ macular hole procedures, automated analysis of both the macular hole geometry and the outer retinal changes may impact a surgeon's understanding of hole closure rate and allow individualized care based on the intraoperative environment (Figure 3). 51,52

The seamless integration of all of these tools combined with emerging OCT technology and cuttingedge visualization systems may create a new paradigm for a comprehensive image-guided surgical feedback platform for ophthalmic surgery. Using OCT-based automated assessment of relative tissue-instrument position, instrument depth information may be able to be provided to the surgeon. Novel therapeutics that demand enhanced precision may require image-assisted delivery or image-guided confirmation of optimal dosing.

Last, the overall value proposition of intraoperative OCT for patient outcomes needs additional validation. Although studies have clearly demonstrated a clear impact on surgical decision, the overall impact on patient outcomes is not clear. Randomized masked prospective trials for disease-specific end points are likely needed to better define the overall value. These studies are currently being planned.

\section{Conclusion}

As OCT has revolutionized the clinical management of ophthalmic diseases, intraoperative OCT is potentially poised as a game-changing technology in the operating room. Over the past few years, significant advances in integrative technology have paved the way to first generation commercial systems. Future platforms will need to prioritize surgeon interaction, image quality, and overall data analysis to the value of this technology. Emerging therapies, such as gene therapy and stem cell therapy, may provide unique opportunities for targeted image-guided delivery with confirmatory imaging 

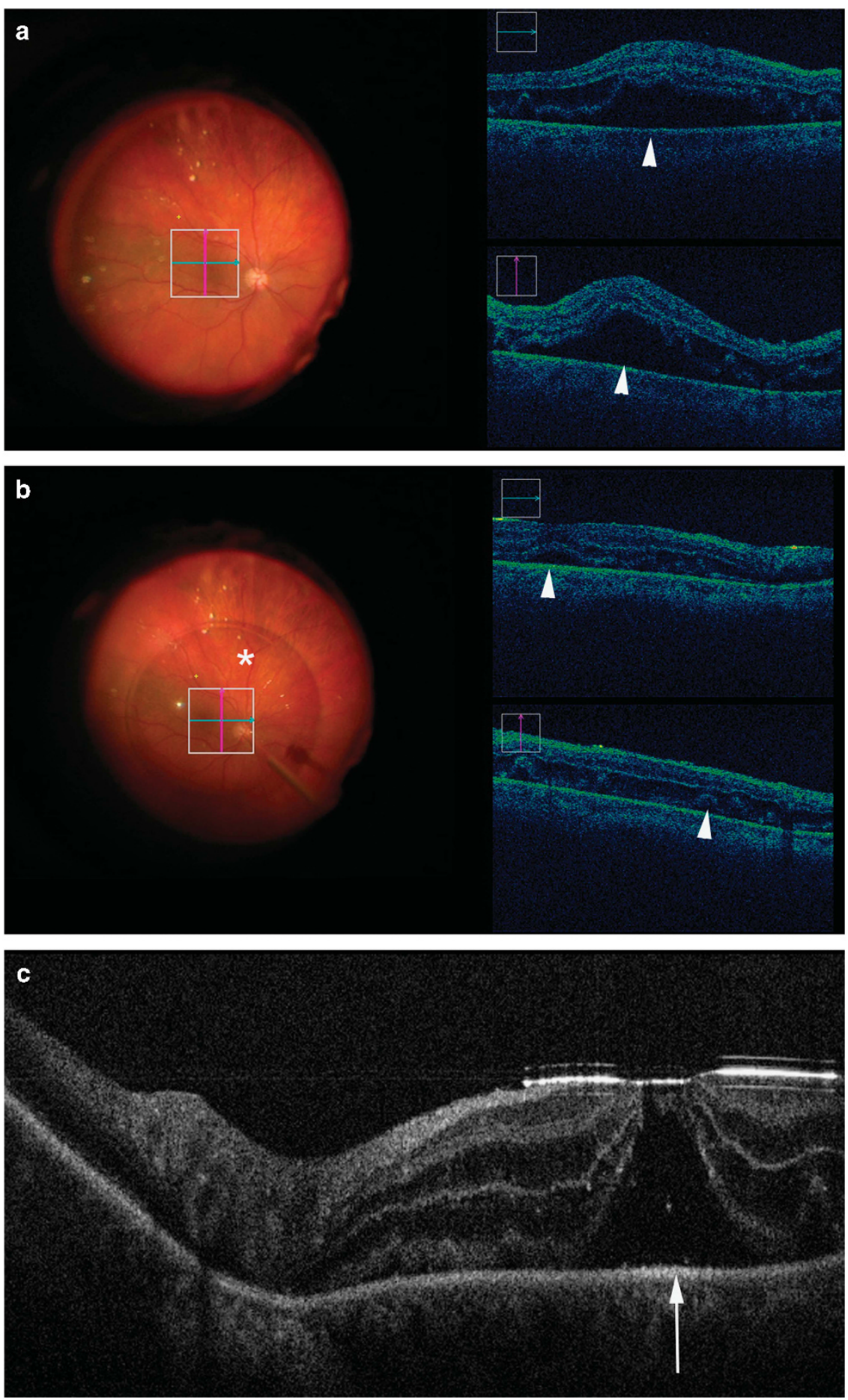

Figure 4 Intraoperative OCT during retinal detachment surgery. (a) First case example prior to perfluorocarbon liquid tamponade revealing significant subretinal fluid (arrowheads) in macula. (b) During perfluorocarbon liquid application (asterisk), subretinal fluid dramatically decreases but is still noted in the submacular area (arrowheads). (c) Second case example following application of perfluorocarbon liquid reveals significant alterations to foveal contour with a potential occult full-thickness macular hole (arrow).

of optimal placement. Immediate surgeon feedback regarding the surgical environment may facilitate the transition to more novel procedures for surgeons. In membrane peeling procedures, intraoperative OCT may maximize surgical efficiency while minimizing unnecessary surgical manipulations. Continuing research and development is still needed for intraoperative OCT but early studies suggest that 
this technology may represent a unique opportunity for individualized enhanced patient care.

\section{Conflict of interest}

JPE: Bioptigen $(C, P)$, Thrombogenics $(C, R)$, Synergetics $(\mathrm{P})$, Genentech $(\mathrm{R})$, Leica $(\mathrm{C})$, Zeiss $(\mathrm{C})$, Alcon $(\mathrm{C})$.

\section{Acknowledgements}

The work was supported by NIH/NEI K23-EY022947-01A1 to (JPE) Ohio Department of Development TECH-13-059 (JPE); Research to Prevent Blindness (Cole Eye Institutional).

\section{References}

1 Kuhn TS. The Structure of Scientific Revolutions. University of Chicago Press: Chicago, IL, USA, 1962.

2 Machemer R, Buettner H, Norton EW, Parel JM. Vitrectomy: a pars plana approach. Trans Am Acad Ophthalmol Otolaryngol 1971; 75(4): 813-820.

3 Machemer R, Parel JM. An improved microsurgical ceilingmounted unit and automated television. Am J Ophthalmol 1978; 85(2): 205-209.

4 Chen TC, Cense B, Pierce MC, Nassif N, Park BH, Yun SH et al. Spectral domain optical coherence tomography: ultrahigh speed, ultra-high resolution ophthalmic imaging. Arch Ophthalmol 2005; 123(12): 1715-1720.

5 Hee MR, Izatt JA, Swanson EA, Huang D, Schuman JS, Lin CP et al. Optical coherence tomography of the human retina. Arch Ophthalmol 1995; 113(3): 325-332.

6 Chavala SH, Farsiu S, Maldonado R, Wallace DK, Freedman $\mathrm{SF}$, Toth CA. Insights into advanced retinopathy of prematurity using handheld spectral domain optical coherence tomography imaging. Ophthalmology 2009; 116(12): 2448-2456

7 Dayani PN, Maldonado R, Farsiu S, Toth CA. Intraoperative use of handheld spectral domain optical coherence tomography imaging in macular surgery. Retina 2009; 29(10): 1457-1468.

8 Ehlers JP, Kernstine K, Farsiu S, Sarin N, Maldonado R, Toth CA. Analysis of pars plana vitrectomy for optic pit-related maculopathy with intraoperative optical coherence tomography: a possible connection with the vitreous cavity. Arch Ophthalmol 2011; 129(11): 1483-1486.

9 Knecht PB, Kaufmann C, Menke MN, Watson SL, Bosch MM. Use of intraoperative fourier-domain anterior segment optical coherence tomography during descemet stripping endothelial keratoplasty. Am J Ophthalmol 2010; 150(3): 360-365.

10 Pichi F, Alkabes M, Nucci P, Ciardella AP. Intraoperative $\mathrm{SD}-\mathrm{OCT}$ in macular surgery. Ophthalmic Surg Lasers Imaging 2012; 43(6 Suppl): S54-S60.

11 Ray R, Baranano DE, Fortun JA, Schwent BJ, Cribbs BE, Bergstrom CS et al. Intraoperative microscope-mounted spectral domain optical coherence tomography for evaluation of retinal anatomy during macular surgery. Ophthalmology 2011; 118(11): 2212-2217.

12 Scorcia V, Busin M, Lucisano A, Beltz J, Carta A, Scorcia G. Anterior segment optical coherence tomography-guided big-bubble technique. Ophthalmology 2013; 120(3): 471-476.
13 Ehlers JP, Dupps WJ, Kaiser PK, Goshe J, Singh RP, Petkovsek D et al. The prospective intraoperative and Perioperative Ophthalmic ImagiNg with Optical CoherEncE TomogRaphy (PIONEER) study: 2-Year Results. Am J Ophthalmol 2014; 158(5): 999-1007.

14 Ehlers JP, Griffith JF, Srivastava SK. Intraoperative optical coherence tomography during vitreoretinal surgery for dense vitreous hemorrhage in the pioneer study. Retina 2015; 35: 2537-2542.

15 Ehlers JP, Petkovsek DS, Yuan A, Singh RP, Srivastava SK. Intrasurgical assessment of subretinal tPA injection for submacular hemorrhage in the PIONEER study utilizing intraoperative OCT. Ophthalmic Surg Lasers Imaging Retina 2015; 46(3): 327-332.

16 Ehlers JP, Tam T, Kaiser PK, Martin DF, Smith GM, Srivastava SK. Utility of intraoperative optical coherence tomography during vitrectomy surgery for vitreomacular traction syndrome. Retina 2014; 34(7): 1341-1346.

17 Ehlers JP, Xu D, Kaiser PK, Singh RP, Srivastava SK. Intrasurgical dynamics of macular hole surgery: an assessment of surgery-induced ultrastructural alterations with intraoperative optical coherence tomography. Retina 2014; 34(2): 213-221.

18 Scott AW, Farsiu S, Enyedi LB, Wallace DK, Toth CA. Imaging the infant retina with a hand-held spectral-domain optical coherence tomography device. Am J Ophthalmol 2009; 147(2): 364-73.

19 Ehlers JP, Tao YK, Farsiu S, Maldonado R, Izatt JA, Toth CA. Integration of a spectral domain optical coherence tomography system into a surgical microscope for intraoperative imaging. Invest Ophthalmol Vis Sci 2011; 52(6): 3153-3159.

20 Tao YK, Ehlers JP, Toth CA, Izatt JA. Intraoperative spectral domain optical coherence tomography for vitreoretinal surgery. Opt Lett 2010; 35(20): 3315-3317.

21 Binder S, Falkner-Radler CI, Hauger C, Matz H, Glittenberg C. Feasibility of intrasurgical spectral-domain optical coherence tomography. Retina 2011; 31(7): 1332-1336.

22 Hirnschall N, Amir-Asgari S, Maedel S, Findl O. Predicting the postoperative intraocular lens position using continuous intraoperative optical coherence tomography measurements. Invest Ophthalmol Vis Sci 2013; 54(8): 5196-5203.

23 Hirnschall N, Norrby S, Weber M, Maedel S, Amir-Asgari S, Findl $\mathrm{O}$. Using continuous intraoperative optical coherence tomography measurements of the aphakic eye for intraocular lens power calculation. Br J Ophthalmol 2015; 99(1): 7-10.

24 Ehlers JP, Tao YK, Farsiu S, Maldonado R, Izatt JA, Toth CA. Visualization of real-time intraoperative maneuvers with a microscope-mounted spectral domain optical coherence tomography system. Retina 2013; 33(1): 232-236.

25 Ehlers JP, Srivastava SK, Feiler D, Noonan AI, Rollins AM, Tao YK. Integrative advances for OCT-guided ophthalmic surgery and intraoperative OCT: microscope integration, surgical instrumentation, and heads-up display surgeon feedback. PloS One 2014; 9(8): e105224.

26 Tao YK, Srivastava SK, Ehlers JP. Microscope-integrated intraoperative OCT with electrically tunable focus and heads-up display for imaging of ophthalmic surgical maneuvers. Biomed Opt Express 2014; 5(6): 1877-1885.

27 Ehlers JP, Goshe J, Dupps WJ, Kaiser PK, Singh RP, Gans R et al. Determination of feasibility and utility of microscopeintegrated optical coherence tomography during ophthalmic Surgery: The DISCOVER Study RESCAN Results. JAMA Ophthalmol 2015; 133(10): 1124-1132. 
28 Ehlers JP, Kaiser PK, Srivastava SK. Intraoperative optical coherence tomography using the RESCAN 700: preliminary results from the DISCOVER study. Br J Ophthalmol 2014; 98 (10): 1329-1332.

29 Steven P, Le Blanc C, Velten K, Lankenau E, Krug M, Oelckers $\mathrm{S}$ et al. Optimizing descemet membrane endothelial keratoplasty using intraoperative optical coherence tomography. JAMA Ophthalmol 2013; 131(9): 1135-1142.

30 Ehlers JP, Kaiser PK, Singh RP, Srivastava SK. The DISCOVER Study 1-Year Results. American Society of Retina Specialists: Vienna, Austria: 2015.

31 Toth CA, Izatt JA. Surgically integrated 4-dimensional, swept-source optical coherence tomography (SSOCT) with heads up display to guide vitreoretinal surgery, in American Society of Retina Specialists, 11-14 July 2015; Vienna, Austria. 2015.

$32 \mathrm{Au}$ J, Goshe J, Dupps Jr WJ, Srivastava SK, Ehlers JP. Intraoperative optical coherence tomography for enhanced depth visualization in deep anterior lamellar keratoplasty from the PIONEER study. Cornea 2015; 34(9): 1039-1043.

33 Cost B, Goshe JM, Srivastava S, Ehlers JP. Intraoperative optical coherence tomography-assisted descemet membrane endothelial keratoplasty in the DISCOVER study. Am J Ophthalmol 2015; 160(3): 430-437.

34 Ehlers JP, Itoh Y, Xu L, Kaiser PK, Singh RP, Srivastava SK. Factors associated with persistent subfoveal fluid and complete macular hole closure in the PIONEER study. Invest Ophthalmol Vis Sci 2014; 56(2): 1141-1146.

35 Ehlers JP, Ohr MP, Kaiser PK, Srivastava SK. Novel microarchitectural dynamics in rhegmatogenous retinal detachments identified with intraoperative optical coherence tomography. Retina 2013; 33(7): 1428-1434.

36 Ehlers JP, Tao YK, Srivastava SK. The value of intraoperative optical coherence tomography imaging in vitreoretinal surgery. Curr Opin Ophthalmol 2014; 25(3): 221-227.

37 Falkner-Radler CI, Glittenberg C, Gabriel M, Binder S. Intrasurgical microscope-integrated spectral domain optical coherence tomography-assisted membrane peeling. Retina 2015; 35(10): 2100-2106.

38 Hahn P, Migacz J, O'Connell R, Maldonado RS, Izatt JA, Toth CA. The use of optical coherence tomography in intraoperative ophthalmic imaging. Ophthalmic Surg Lasers Imaging 2011; 42(Suppl): S85-S94.

39 Juthani VV, Goshe JM, Srivastava SK, Ehlers JP. Association between transient interface fluid on intraoperative OCT and textural interface opacity after DSAEK surgery in the PIONEER study. Cornea 2014; 33(9): 887-892.

40 Matz H, Binder S, Glittenberg C, Scharioth G, Findl O, Hirnschall $\mathrm{N}$ et al. Intraoperative Applications of OCT in Ophthalmic Surgery. Biomed Tech 2012; 57(Suppl 1).
41 Saad A, Guilbert E, Grise-Dulac A, Sabatier P, Gatinel D. Intraoperative OCT-assisted DMEK: 14 aonsecutive cases. Cornea 2015; 34(7): 802-807.

42 De Benito-Llopis L, Mehta JS, Angunawela RI, Ang M, Tan DT. Intraoperative anterior segment optical coherence tomography: a novel assessment tool during deep anterior lamellar keratoplasty. Am J Ophthalmol 2014; 157(2): 334-341.

43 Diaz R, Randolph J, Sigler E, Calzada J. Intraoperative grasp site correlation with morphologic changes in retinal nerve fiber layer after internal limiting membrane peeling. Ophthalmic Surg Lasers Imaging Retina 2014; 45(1): 45-49.

44 Hahn P, Carrasco-Zevallos O, Cunefare D, Migacz J, Farsiu S, Izatt JA et al. Intrasurgical human retinal imaging with manual instrument tracking using a microscope-integrated spectraldomain optical coherence tomography device. Transl Vis Sci Technol 2015; 4(4): 1.

45 Hahn P, Migacz J, O'Donnell R, Day S, Lee A, Lin P et al. Preclinical evaluation and intraoperative human retinal imaging with a high-resolution microscope-integrated spectral domain optical coherence tomography device. Retina 2013; 33(7): 1328-1337.

46 Ehlers JP, Han J, Petkovsek D, Kaiser PK, Singh RP, Srivastava SK. Membrane Peeling- Induced Retinal Alterations on Intraoperative OCT in Vitreomacular Interface Disorders from the PIONEER Study. Invest Ophthalmol Vis Sci 2015; 56: 7324-7330.

47 El-Haddad MT, Tao YK. Automated stereo vision instrument tracking for intraoperative OCT guided anterior segment ophthalmic surgical maneuvers. Biomed Opt Express 2015; 6(8): 3014-3031.

48 Ehlers JP, McNutt S, Dar S, Tao YK, Srivastava SK. Visualisation of contrast-enhanced intraoperative optical coherence tomography with indocyanine green. Br J Ophthalmol 2014; 98(11): 1588-1591.

49 Ehlers JP, McNutt SA, Kaiser PK, Srivastava SK. Contrastenhanced intraoperative optical coherence tomography. Br J Ophthalmol 2013; 97(11): 1384-1386.

50 Xu D, Dupps WJ, Srivastava S, Ehlers JP. Automated volumetric analysis of interface fluid in descemet stripping automated endothelial keratoplasty utilizing intraoperative optical coherence tomography. Invest Ophthalmol Vis Sci 2014; 55(9): 5610-5615.

51 Itoh Y, Vasanji A, Ehlers JP. Volumetric ellipsoid zone mapping for enhanced visualisation of outer retinal integrity with optical coherence tomography. Br J Ophthalmol 2015; e-pub ahead of print 22 July 2015; doi:10.1136/bjophthalmol2015-307105.

52 Xu D, Yuan A, Kaiser PK, Srivastava SK, Singh RP, Sears JE et al. A novel segmentation algorithm for volumetric analysis of macular hole boundaries identified with optical coherence tomography. Invest Ophthalmol Vis Sci 2013; 54(1): 163-169. 